\title{
Pure detection of the acoustic spin pumping in Pt/YIG/PZT structures
}

\author{
Ken-ichi Uchida ${ }^{\mathrm{a}, \mathrm{b}, *}$, Zhiyong Qiu ${ }^{\mathrm{c}}$, Takashi Kikkawa ${ }^{\mathrm{a}}$, Eiji Saitoha,c,d,e \\ ${ }^{a}$ Institute for Materials Research, Tohoku University, Sendai 980-8577, Japan \\ ${ }^{b}$ PRESTO, Japan Science and Technology Agency, Saitama 332-0012, Japan \\ ${ }^{c}$ WPI Advanced Institute for Materials Research, Tohoku University, Sendai 980-8577, \\ Japan \\ ${ }^{d}$ Advanced Science Research Center, Japan Atomic Energy Agency, Tokai 319-1195, \\ Japan \\ ${ }^{e}$ CREST, Japan Science and Technology Agency, Tokyo 102-0076, Japan
}

\begin{abstract}
The acoustic spin pumping (ASP) stands for the generation of a spin voltage from sound waves in a ferromagnet/paramagnet junction. In this letter, we propose and demonstrate a method for pure detection of the ASP, which enables the separation of sound-wave-driven spin currents from the spin Seebeck effect due to the heating of a sample caused by a sound-wave injection. Our demonstration using a Pt/YIG/PZT sample shows that the ASP signal in this structure measured by a conventional method is considerably offset by the heating signal and that the pure ASP signal is one order of magnitude greater than that reported in the previous study.

Keywords: A. insulators, A. magnetically ordered materials, D. phonons, D. spin dynamics
\end{abstract}

PACS: 85.75.-d, 43.35.+d, 63.20.kk

*Tel.: +8122 2152023 .

Email address: kuchida@imr.tohoku.ac.jp (Ken-ichi Uchida)

Preprint submitted to Solid State Communications

August 14, 2013

(C) 2013. This manuscript version is made available under the Elsevier user license 
The emerging field called spin mechanics is a branch of spintronics that focuses on the interaction among spin, charge, and mechanical dynamics. A main purpose of spin mechanics is to develop novel spin-related phenomena based on mechanical motion, vibration, and magnetoelastic coupling $(1 ; 2)$. Recently, spin-mechanic effects have been receiving increasing attention both theoretically and experimentally, and important topics in this field include the conversion between spin and mechanical angular momenta (3), mechanical generation of spin currents in rotating or accelerating nonmagnetic systems $(4 ; 5 ; 6)$, elastically driven ferromagnetic resonance (FMR) $(7 ; 8 ; 9)$, and the acoustic spin pumping (ASP) $(10 ; 11 ; 12 ; 13)$. We reported the observation of the ASP in 2011 and showed that this phenomenon enables the generation of a spin voltage as a result of a sound-wave injection into a system comprising a ferromagnet. The ASP has been operated by sound waves of several $\mathrm{MHz}$ frequencies ( $<10 \mathrm{MHz}$, far below the FMR frequency) as a consequence of the energy transfer from sound waves to spin waves, or magnons, in a ferromagnet, while the resonant spin-current generation based on the elastically driven FMR has been operated by surface acoustic waves of several $\mathrm{GHz}$ frequencies (9).

Figure 1(a) shows a schematic illustration of the sample system for measuring the ASP used in the previous study (10;12). The sample consists of a ferrimagnetic insulator (FI) slab with a paramagnetic metal (PM) film attached to the top surface of the FI. This PM/FI junction is fixed on a piezoelectric actuator; when an $\mathrm{AC}$ voltage is applied between the top and bottom electrodes of the actuator, it vibrates in the thickness direction and injects longitudinal sound waves into the attached FI slab (see Fig. 1(a)). 
In the $\mathrm{PM} / \mathrm{FI} /$ piezoelectric-actuator system, when the injected sound wave modulates the magnon distribution function (effective magnon temperature (14)) in the FI slab via the magnon-phonon interaction, it induces a spin current in the Pt layer with the spatial direction $\mathbf{J}_{\mathrm{s}}$ and the spin-polarization vector $\boldsymbol{\sigma}(10 ; 11 ; 12 ; 13)$. This sound-wave-driven spin current is converted into a DC electric field $\mathbf{E}_{\text {ISHE }}$ due to the inverse spin Hall effect (ISHE) $(15 ; 16 ; 17 ; 18 ; 19 ; 20)$ in the PM layer. When the magnetization of the FI slab $\left(\| \boldsymbol{\sigma}\right.$ in the Pt film) is along the $y$ direction, $\mathbf{E}_{\mathrm{ISHE}}$ is generated along the $x$ direction because of the following ISHE symmetry (see Fig. 1(a)):

$$
\mathbf{E}_{\mathrm{ISHE}} \propto \mathbf{J}_{\mathrm{S}} \times \boldsymbol{\sigma}
$$

Therefore, by measuring $\mathbf{E}_{\text {ISHE }}$, we can detect the ASP electrically. The ISHE induced by the ASP has been observed in $\mathrm{Pt} / \mathrm{Y}_{3} \mathrm{Fe}_{5} \mathrm{O}_{12}$ (YIG) structures attached to a piezoelectric polyvinylidene-fluoride (PVDF) film or a leadzirconate-titanate $\left(\mathrm{Pb}(\mathrm{Zr}, \mathrm{Ti}) \mathrm{O}_{3}: \mathrm{PZT}\right)$ ceramic $(10 ; 12)$.

In Fig. 2, we show the voltage $V$ between the ends of a $\mathrm{Pt}$ film in the conventional Pt/YIG/PZT structure as a function of the frequency $f$ of the AC voltage applied to the PZT ceramic $(10 ; 12)$. In the $V$ spectrum, a dip structure was found to appear around the piezoelectric resonance frequency $f_{\text {piezo }}$ of the PZT (compare Fig. 2 and its inset). As shown in the previous literature on the ASP, the $V$-dip signal originates from the ISHE induced by the resonant sound-wave injection under the piezoelectric resonance condition $(10 ; 12)$.

To observe the ASP, it is important to separate the sound-wave-driven signals from extrinsic heating effects; when the piezoelectric actuator is heated due to the applied AC voltage, a temperature gradient along the $z$ direction 
in the PM/FI structure is also formed and induces the ISHE via the longitudinal spin Seebeck effect (LSSE) $(13 ; 21 ; 22 ; 23 ; 24 ; 25 ; 26 ; 27 ; 28 ; 29)$ (see Fig. 1(b)). In the conventional Pt/YIG/PVDF structures, we distinguished the ASP signal from the LSSE by means of the difference in the spectrum shapes and signal signs between ASP and LSSE (10;12), where the ASP (the LSSE due to the heating of the actuator) exhibits the negative (positive) ISHE voltage in the configuration shown in Fig. 1. In contrast, in the $\mathrm{Pt} / \mathrm{YIG} / \mathrm{PZT}$ structures, since the PZT ceramic is strongly heated under the piezoelectric resonance condition and the shapes of the voltage and heating spectra are similar to each other, one has to judge the origin of voltage only from the signal sign, a situation making it difficult to quantify the pure ASP signal. In this letter, we propose and demonstrate a method for the pure detection of the ASP, which is applicable to various sample systems irrespective of the species of piezoelectric actuators.

The basic idea of the pure ASP detection method is very simple; it is based on the measurement of a sound-wave-driven voltage with controlling the temperature difference between the top and bottom of the PM/FI structure. If the ISHE voltage appears even in the absence of the temperature gradient, the signal is due entirely to the ASP induced by the sound-wave injection. To demonstrate this method, the Pt/YIG sample and the PZT actuator are sandwiched between two aluminum-nitride plates with high thermal conductivity $(\sim 160 \mathrm{~W} / \mathrm{mK})$. Here, the upper aluminum-nitride plate (AlN1) is thermally connected to a heat bath and the lower one (AlN2) is placed on a thermoelectric Peltier module (see the inset to Fig. 3(a)). The bottom of the Peltier module is also thermally connected to the heat bath. 
The temperature of the heat bath is fixed at $300 \mathrm{~K}$. In this setup, by applying a charge current to the Peltier module, we can change the temperature of the AlN2, enabling the control of the temperature gradient in the Pt/YIG sample, where the temperature difference between the AlN1 plate and the bottom of the YIG slab is measured with a differential thermocouple. To detect the pure ASP signal, we measured a DC electric voltage difference $V$ between the ends of the Pt layer while fixing the frequency $f$ of the AC voltage at the piezoelectric resonance frequency $f_{\text {piezo }}(=3.6 \mathrm{MHz})$ of the PZT. During the measurements, an in-plane external magnetic field $\mathbf{H}$ with the magnitude $H$ was applied perpendicular to the inter-electrode direction (see the inset to Fig. 3(a)). All the measurements were performed in a high vacuum.

Figure 3(a) shows that the temperature-difference $\Delta T$ dependence of $V$ at $H=1 \mathrm{kOe}$ for various values of the AC-voltage amplitude $V_{\mathrm{pp}}$ applied to the PZT. We confirmed that the magnitude of $V$ varies linearly with respect to the temperature difference due to the LSSE. Importantly, when finite sound waves are applied to the sample, the $V$ signal offset at $\Delta T=$ $0 \mathrm{~K}, V_{0}$, was found to monotonically decrease with increasing $V_{\mathrm{pp}}$. Since the LSSE voltage and other thermoelectric artifacts, such as conventional Nernst-Ettingshausen effects (30) in the Pt layer, disappear at $\Delta T=0 \mathrm{~K}$, this negative $V$ offset is due purely to the sound waves injected into the $\mathrm{Pt} /$ YIG sample. We also confirmed that $V_{0}$ is proportional to the square of the AC-voltage amplitude $V_{\mathrm{pp}}^{2}$, indicating the proportionality between $V_{0}$ and the sound-wave intensity in the YIG slab (see Figs. 3(b) and 3(c)), where we assume that the vibration amplitude of the piezoelectric actuator $\Delta d_{\text {piezo }}$ 
is proportional to $V_{\mathrm{pp}}$. As shown in Fig. $3(\mathrm{~d})$, the $V$ signal at $\Delta T=0 \mathrm{~K}$ is reversed when the $\mathbf{H}$ direction is reversed (31). These results are consistent with the characteristic of the ISHE induced by the ASP.

Notably, the magnitude of the ASP voltage in the Pt/YIG/PZT sample in the present configuration is $\left|V_{0} / V_{\mathrm{pp}}^{2}\right| \sim 6.3 \times 10^{-8} \mathrm{~V}^{-1}$ at $f_{\text {piezo }}=3.6 \mathrm{MHz}$, which is one order of magnitude greater than that in the conventional configuration: $\sim 0.8 \times 10^{-8} \mathrm{~V}^{-1}$ (compare Figs. 2 and 3 ). This result means that the ASP signal in the Pt/YIG/PZT sample measured in the conventional configuration is considerably offset by the LSSE voltage and that the actual spin-current magnitude induced by the ASP in this sample is much greater than that estimated from the previous data. Here, the ASP-induced spin-current density $j_{\mathrm{s}}$ normalized by $\Delta d_{\text {piezo }}^{2}$ can be described as $(19 ; 20)$

$$
\frac{j_{\mathrm{s}}}{\Delta d_{\text {piezo }}^{2}}=\left(\frac{\hbar}{2 e}\right) \frac{d_{\mathrm{PM}} \sigma_{\mathrm{PM}}}{l_{\mathrm{PM}} \theta_{\mathrm{SH}} \lambda_{\mathrm{PM}} \tanh \left(d_{\mathrm{PM}} / 2 \lambda_{\mathrm{PM}}\right)} \frac{V_{0}}{D_{\text {piezo }}^{2} V_{\mathrm{pp}}^{2}},
$$

where $e$ is the electron charge, $\hbar$ is the Planck constant divided by $2 \pi$, $D_{\text {piezo }} \equiv \Delta d_{\text {piezo }} / V_{\mathrm{pp}}$, and $l_{\mathrm{PM}}, d_{\mathrm{PM}}, \sigma_{\mathrm{PM}}, \theta_{\mathrm{SH}}$, and $\lambda_{\mathrm{PM}}$ are the length, thickness, electric conductivity, spin Hall angle, and spin diffusion length of PM, respectively. In the Pt/YIG/PZT structure in the present configuration, by using $l_{\mathrm{PM}}=6 \mathrm{~mm}, d_{\mathrm{PM}}=10 \mathrm{~nm}, \sigma_{\mathrm{PM}}=3.1 \times 10^{6} \mathrm{~S} / \mathrm{m}$, and $D_{\text {piezo }} \sim 0.5 \mathrm{~nm} / \mathrm{V}$ (see the inset to Fig. 2) and by assuming $\theta_{\mathrm{SH}}=0.01$ and $\lambda_{\mathrm{PM}}=7.7 \mathrm{~nm}(20),\left|j_{\mathrm{s}}\right|$ per 1-nm thickness-vibration amplitude is estimated to be $\sim 1 \times 10^{-11} \mathrm{~J} / \mathrm{m}^{2}$, which corresponds to $\left|\widetilde{j}_{\mathrm{s}}\right| \sim 3 \times 10^{4} \mathrm{~A} / \mathrm{m}^{2}$ with $\widetilde{j}_{\mathrm{s}}=(2 e / \hbar) j_{\mathrm{s}}$. When we assume the recently-reported values of the spin Hall angle and spin diffusion length for Pt: $\theta_{\mathrm{SH}}=0.12$ and $\lambda_{\mathrm{PM}}=1.3 \mathrm{~nm}$ (32), $\left|j_{\mathrm{s}}\right|$ and $\left|\widetilde{j}_{\mathrm{s}}\right|$ per 1-nm thickness-vibration amplitude are estimated to be $\sim 3 \times 10^{-12} \mathrm{~J} / \mathrm{m}^{2}$ and $\sim 8 \times 10^{3} \mathrm{~A} / \mathrm{m}^{2}$, respectively. 
In conclusion, we reported the pure detection of the acoustic spin pumping (ASP) in the Pt/YIG/PZT structure. The experimental results show that the pure ISHE voltage induced by the ASP in the Pt/YIG/PZT structure is one order of magnitude greater than that reported in the previous study. The experimental method demonstrated here enables the complete separation of the ASP signals from the heating effects, such as the conventional spin Seebeck effects and Nernst-Ettingshausen effects. This method allows various piezoelectric materials including PZT to be utilized for the systematic investigation and quantitative estimation of the ASP.

\section{Acknowledgments}

The authors thank S. Maekawa, H. Adachi, and H. Nakayama for valuable discussions. This work was supported by PRESTO-JST "Phase Interfaces for Highly Efficient Energy Utilization", CREST-JST "Creation of Nanosystems with Novel Functions through Process Integration", Grant-in-Aid for Young Scientists (A) (25707029) from MEXT, Japan, Grant-in-Aid for Scientific Research (A) (24244051) from MEXT, Japan, LC-IMR of Tohoku University, The Murata Science Foundation, The Mazda Foundation, and The Sumitomo Foundation.

\section{References}

[1] H. Matthews, R. C. LeCraw, Phys. Rev. Lett. 8 (1962) 397.

[2] A. K. Ganguly, K. L. Davis, D. C. Webb, C. Vittoria, J. Appl. Phys. 47 (1976) 2696. 
[3] G. Zolfagharkhani, A. Gaidarzhy, P. Degiovanni, S. Kettemann, P. Fulde, P. Mohanty, Nature Nanotech. 3 (2008) 720.

[4] M. Matsuo, J. Ieda, E. Saitoh, S. Maekawa, Phys. Rev. Lett. 106 (2011) 076601.

[5] M. Matsuo, J. Ieda, E. Saitoh, S. Maekawa, Phys. Rev. B 84 (2011) 104410.

[6] M. Matsuo, J. Ieda, K. Harii, E. Saitoh, S. Maekawa, Phys. Rev. B 87 (2013) 180402(R).

[7] S. Maekawa, M. Tachiki, AIP Conf. Proc. 29 (1976) 542.

[8] M. Weiler, L. Dreher, C. Heeg, H. Huebl, R. Gross, M. S. Brandt, S. T. B. Goennenwein, Phys. Rev. Lett. 106 (2011) 117601.

[9] M. Weiler, H. Huebl, F. S. Goerg, F. D. Czeschka, R. Gross, S. T. B. Goennenwein, Phys. Rev. Lett. 108 (2012) 176601.

[10] K. Uchida, H. Adachi, T. An, T. Ota, M. Toda, B. Hillebrands, S. Maekawa, E. Saitoh, Nature Mater. 10 (2011) 737.

[11] K. Uchida, T. An, Y. Kajiwara, M. Toda, E. Saitoh, Appl. Phys. Lett. 99 (2011) 212501.

[12] K. Uchida, H. Adachi, T. An, H. Nakayama, M. Toda, B. Hillebrands, S. Maekawa, E. Saitoh, J. Appl. Phys. 111 (2012) 053903.

[13] H. Adachi, K. Uchida, E. Saitoh, S. Maekawa, Rep. Prog. Phys. 76 (2013) 036501. 
[14] J. Xiao, G. E. W. Bauer, K. Uchida, E. Saitoh, S. Maekawa, Phys. Rev. B 81 (2010) 214418.

[15] A. Azevedo, L. H. Vilela Leao, R. L. Rodriguez-Suarez, A. B. Oliveira, S. M. Rezende, J. Appl. Phys. 97 (2005) $10 \mathrm{C} 715$.

[16] E. Saitoh, M. Ueda, H. Miyajima, G. Tatara, Appl. Phys. Lett. 88 (2006) 182509.

[17] S. O. Valenzuela, M. Tinkham, Nature 442 (2006) 176.

[18] T. Kimura, Y. Otani, T. Sato, S. Takahashi, S. Maekawa, Phys. Rev. Lett. 98 (2007) 156601.

[19] K. Ando, S. Takahashi, J. Ieda, Y. Kajiwara, H. Nakayama, T. Yoshino, K. Harii, Y. Fujikawa, M. Matsuo, S. Maekawa, E. Saitoh, J. Appl. Phys. 109 (2011) 103913.

[20] H. Nakayama, K. Ando, K. Harii, T. Yoshino, R. Takahashi, Y. Kajiwara, K. Uchida, Y. Fujikawa, E. Saitoh, Phys. Rev. B 85 (2012) 144408.

[21] K. Uchida, H. Adachi, T. Ota, H. Nakayama, S. Maekawa, E. Saitoh, Appl. Phys. Lett. 97 (2010) 172505.

[22] K. Uchida, T. Nonaka, T. Ota, E. Saitoh, Appl. Phys. Lett. 97 (2010) 262504 .

[23] K. Uchida, T. Ota, H. Adachi, J. Xiao, T. Nonaka, Y. Kajiwara, G. E. W. Bauer, S. Maekawa, E. Saitoh, J. Appl. Phys. 111 (2012) 103903. 
[24] A. Kirihara, K. Uchida, Y. Kajiwara, M. Ishida, Y. Nakamura, T. Manako, E. Saitoh, S. Yorozu, Nature Mater. 11 (2012) 686.

[25] D. Qu, S. Y. Huang, J. Hu, R. Wu, C. L. Chien, Phys. Rev. Lett. 110 (2013) 067206.

[26] T. Kikkawa, K. Uchida, Y. Shiomi, Z. Qiu, D. Hou, D. Tian, H. Nakayama, X.-F. Jin, E. Saitoh, Phys. Rev. Lett. 110 (2013) 067207.

[27] D. Meier, T. Kuschel, L. Shen, A. Gupta, T. Kikkawa, K. Uchida, E. Saitoh, J.-M. Schmalhorst, G. Reiss, Phys. Rev. B 87 (2013) 054421.

[28] R. Ramos, T. Kikkawa, K. Uchida, H. Adachi, I. Lucas, M. H. Aguirre, P. Algarabel, L. Morellon, S. Maekawa, E. Saitoh, M. R. Ibarra, Appl. Phys. Lett. 102 (2013) 072413.

[29] K. Uchida, T. Nonaka, T. Kikkawa, Y. Kajiwara, E. Saitoh, Phys. Rev. B 87 (2013) 104412.

[30] H. B. Callen, Phys. Rev. 73 (1948) 1349.

[31] The shape of the $V-H$ curves observed here is similar to that of the LSSE in a single-crystalline YIG slab covered with a Pt film (Ref. 21). The different shapes between the $V-H$ curves in the present data (Fig. 3(d)) and the previous ASP data (Refs. 10 and 12) are due to different digital-filter parameters of a nanovoltmeter (Keithley 2182A); since the ASP signals in the previous experiments are very small, we applied a smoothing filter in order to average out the noise, while the $V$ - $H$ curves shown in this paper are raw data. 
[32] H. Jiao, G. E. W. Bauer, Phys. Rev. Lett. 110 (2013) 217602. 
Figure 1: Schematic illustrations of the acoustic spin pumping (ASP, (a)) and the longitudinal spin Seebeck effect (LSSE, (b)) in a paramagnetic metal/ferrimagnetic insulator junction. $\mathbf{E}_{\mathrm{ISHE}}$ and $\mathbf{M}$ denote the electric field generated by the inverse spin Hall effect and the magnetization vector, respectively. The ASP (LSSE) generates a spin voltage as a result of a sound-wave injection (a temperature gradient $\nabla T$ ).

Figure 2: The frequency $f$ dependence of the voltage $V$ in the Pt/YIG/PZT sample at $V_{\mathrm{pp}}=10 \mathrm{~V}$ and $H=1 \mathrm{kOe}$ in the conventional experimental configuration $(10 ; 12)$. The PZT ceramic used here is of a $10 \times 3 \times 0.6 \mathrm{~mm}^{3}$ rectangular shape. The piezoelectric resonance frequency of the PZT ceramics is $3.6 \mathrm{MHz}$. The inset shows the $f$ dependence of the thickness-vibration amplitude $\Delta d_{\text {piezo }}$ of the PZT ceramic due to the applied AC voltage, measured by means of a laser Doppler vibrometry. During the laser Doppler measurements, the PZT ceramic was fixed on a glass-epoxy plate with silver paste, where the top surface of the glass-epoxy plate is coated with a thin $\mathrm{Cu}$ electrode. 
Figure 3: (a) The temperature-difference $\Delta T$ dependence of $V$ in the Pt/YIG/PZT sample at $f=3.6 \mathrm{MHz}$ and $H=1 \mathrm{kOe}$ for various values of the AC-voltage amplitude $V_{\mathrm{pp}}$ (peakto-peak voltage) in the present experimental configuration. The Pt/YIG sample consists of a single-crystalline YIG slab with a Pt film attached to the (100) surface of the YIG. The length, width, and thickness of the YIG slab (Pt film) are $6 \mathrm{~mm}(6 \mathrm{~mm}), 2 \mathrm{~mm}(2$ $\mathrm{mm})$, and $1 \mathrm{~mm}(10 \mathrm{~nm})$, respectively. The PZT ceramics used here is the same as that used for the experiments in Fig. 2. The top and bottom electrodes of the PZT ceramics were attached to the bottom of the YIG slab and the top of the AlN2 plate with silver paste, respectively. The black and dark-red curves show the differential thermocouple for measuring $\Delta T$, which consists of two T-type thermocouples differentially connected to each other. (b),(c) $V_{\mathrm{pp}}$ and $V_{\mathrm{pp}}^{2}$ dependences of $V_{0}$ in the Pt/YIG/PZT sample at $f=3.6 \mathrm{MHz}$ and $H=1 \mathrm{kOe}$. Here, $V_{0}$ is defined as the zero-temperature-difference intercept of the linear fit line of the measured $\Delta T$ - $V$ data (see (a)). (d) $H$ dependence of $V$ in the Pt/YIG/PZT sample at $\Delta T=0 \mathrm{~K}$ for various values of $V_{\mathrm{pp}}$. 


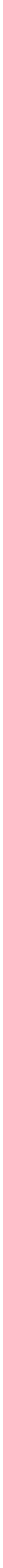

Figure2

.

.
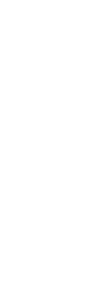

.

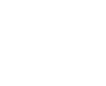
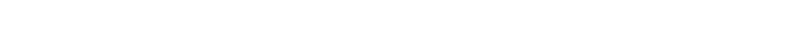
(a) 3

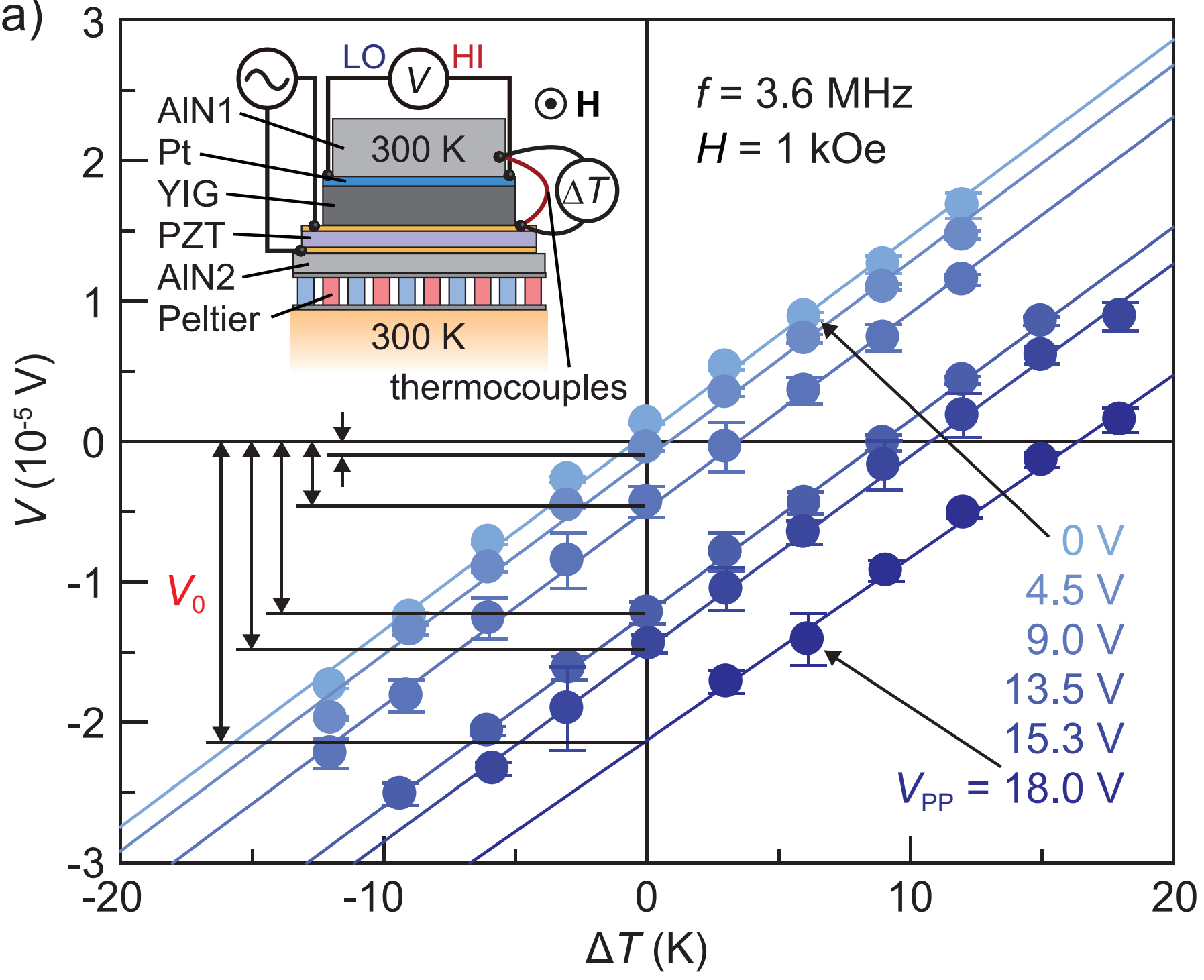

(b)
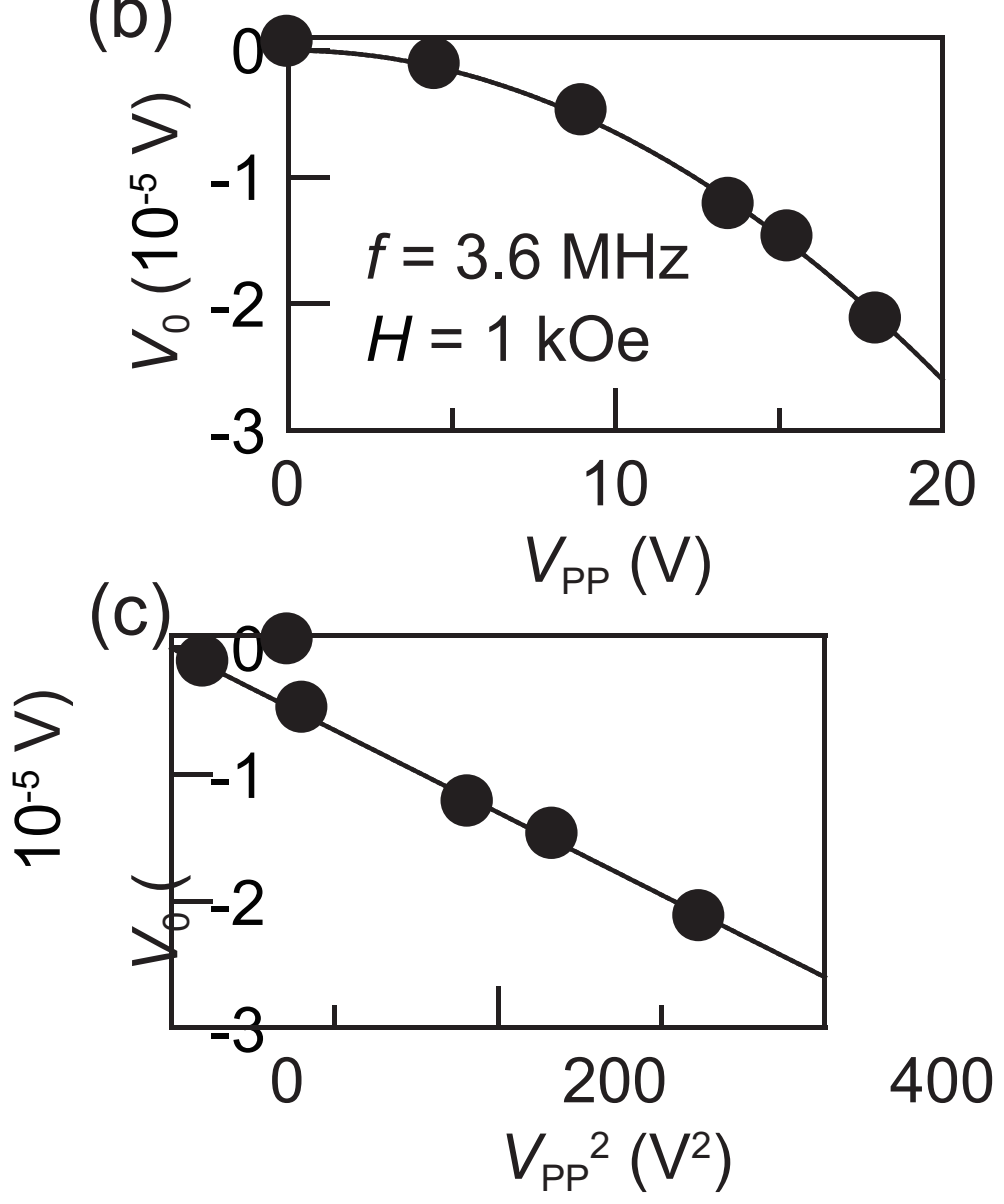

(d)

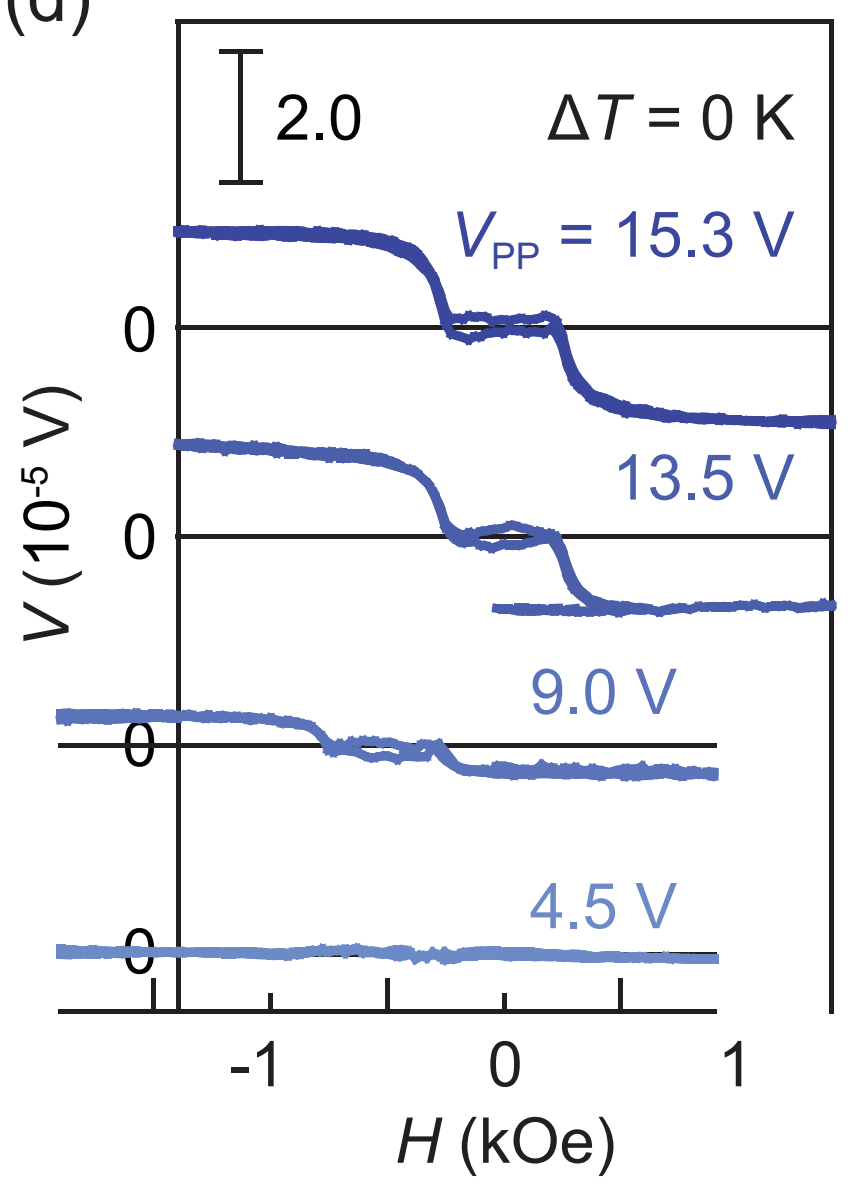

